\title{
Fisiopatologia e desenvolvimento do diabetes mellitus tipo 3 e sua relação com a doença de Alzheimer
}

\author{
Pathophysiology and development of type 3 diabetes mellitus and its relationship with Alzheimer's \\ disease
}

DOI: $10.37111 /$ braspenj. 2020354015

Giúlia Jäger Maximowicz de Oliveira

Camila Luisa Roda Cichacewski

Carolina Fantin Carneiro ${ }^{3}$

Leticia Fuganti Campos ${ }^{4}$

Antônio Carlos Ligocki Campos ${ }^{5}$

\section{Unitermos:}

Doença de Alzheimer. Diabetes mellitus. Resistência à insulina.

\section{Keywords:}

Alzheimer disease. Diabetes mellitus. Insulin resistance.

\section{Endereço de correspondência:}

Leticia Fuganti Campos

NUTROPAR

Rua Bruno Filgueira, 2495 - Bigorrilho - Curitiba, PR, Brasil - CEP: 80710-530

Email: le_campos@hotmail.com

\section{Submissão}

28 de setembro de 2020

Aceito para publicação

11 de dezembro de 2020

\begin{abstract}
RESUMO
Introdução: A doença de Alzheimer (DA) se caracteriza pela deterioração da função cognitiva global, de maneira progressiva e persistente, que resulta em comprometimento das funções corticais. Há alguns anos, a relação entre a DA e o diabetes mellitus tipo 2 (DM2) vem sendo estudada, resultando no termo diabetes mellitus tipo 3 (DM3). Método: Trata-se de uma revisão de literatura, em que foi realizada a busca de artigos publicados, nos últimos 5 anos, nas bases de dados Medline e PubMed, com a utilização dos descritores, na língua portuguesa, inglesa e espanhola: doença de Alzheimer, resistência à insulina e diabetes mellitus tipo 3. Resultados: Para análise foram selecionados 12 artigos, sendo 10 revisões de literatura e 2 estudos originais. Tais trabalhos exploraram a relação celular e molecular entre a DA e a resistência à insulina, bem como possíveis mecanismos patogênicos, o papel da insulina no cérebro, fatores ambientais vinculados à DA e intervenções dietéticas para evitar a neurodegeneração. Conclusão: A relação entre DA e o DM2 se deve à ação de diversos mecanismos, como o metabolismo lipídico, o metabolismo da insulina e agentes relacionados ao seu funcionamento, como o alelo ApoE $\varepsilon 4$, o

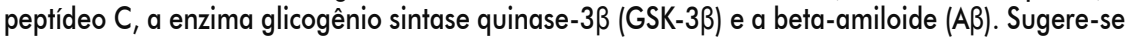
que as diversas alterações, principalmente no metabolismo da insulina, podem prejudicar a função neurocognitiva e desencadear a DA. Estudos futuros são necessários para analisar o contexto do DM3 e encontrar possíveis tratamentos que atenuem a progressão da DA e promovam qualidade de vida aos pacientes.
\end{abstract}

\section{ABSTRACT}

Introduction: Alzheimer's disease (AD) is characterized by a progressive and persistent deterioration of the whole cognitive function, which results in an impaired cortical function. Some years ago, the connection between $A D$ and type 2 diabetes has been studied, resulting in the term type 3 diabetes (T3D). Methods: This is a literature review, a search for articles published in the last 5 years in the Medline and PubMed databases was performed, using the descriptor: Alzheimer disease, diabetes, insulin resistance. Results: For analysis, 12 articles were selected, with 10 literature reviews and 2 original studies. Among those who explored the cellular and molecular relationship between $A D$ and insulin resistance, possible pathogenic mechanisms, the role of insulin in the brain, environmental factors linked to $A D$ and dietary interventions to prevent neurodegeneration. Conclusion: The relation between $A D$ and type 2 diabetes is due several mechanisms such as lipid metabolism, insulin metabolism and agents related to its functioning, like the ApoE\&4, C-peptide, the glycogen synthase kinase $3 \beta$ (GSK-3ß) and the amyloid-beta (A $\beta)$. It is suggested that several changes, mainly in insulin metabolism, can impair neurocognitive function and trigger $A D$. Future studies are needed to analyze the context of T3D and find possible treatments that attenuate the $A D$ progression and promote quality of life for the patients.

1. Nutricionista, pós-graduação em Nutrição clínica e terapia nutricional pela Universidade Positivo, Mestranda em Alimentação e Nutrição pela Universidade Federal do Paraná, Curitiba, PR, Brasil.

2. Nutricionista, pós-graduação em Nutrição clínica e terapia nutricional pela Universidade Positivo, Mestre em Ciências da Saúde pela Pontifícia Universidade Católica do Paraná, Nutricionista clínica do Hospital Santa Casa de Curitiba, Curitiba, PR, Brasil.

3. Nutricionista, pós-graduação em Nutrição clínica e terapia nutricional pela Universidade Positivo, Proprietária/nutricionista da loja Mundo Verde, Água Verde, Curitiba, PR, Brasil.

4. Nutricionista e educadora em diabetes. Pós-graduação em Nutrição Clínica pelo Ganep e em Educação em Diabetes pela UNIP. Especialista pela Sociedade Brasileira de Nutrição Parenteral e Enteral. Mestre pela Faculdade de Medicina da Universidade de São Paulo (FMUSP), Doutora em Medicina (Clínica Cirúrgica) pela Universidade Federal do Paraná, Docente em programas de pós-graduação e nutricionista clínica e sócia da NUTROPAR, Curitiba, PR, Brasil.

5. Cirurgião gastroenterologista, Mestre e Doutor em Clínica Cirúrgica pela Universidade Federal do Paraná, Titular da Cadeira 56 da Academia Paranaense de Medicina, Professor Titular e Coordenador do Programa de Pós-Graduação em Clínica Cirúrgica da Universidade Federal do Paraná, Curitiba, PR, Brasil. 


\section{INTRODUÇÃO}

A demência é uma síndrome de natureza crônica e progressiva da função cognitiva, que afeta memória, pensamento, orientação, compreensão, cálculo, capacidade de aprendizagem, linguagem e julgamento'. Em 2017, estimou-se que 50 milhões de pessoas em todo o mundo apresentavam demência e quase $60 \%$ viviam em países de baixa e média renda. Essa prevalência é prevista para dobrar a cada 20 anos e atingir 75 milhões até 2030.

A doença de Alzheimer (DA) é a forma mais comum de demência e representa cerca de $60 \%$ a $70 \%$ dos casos $^{2}$. É caracterizada como distúrbio neurodegenerativo progressivo, que possui múltiplas cascatas de sinalização ${ }^{3}$. Dentre os vários mecanismos que estão envolvidos no processo da doença, destaca-se o estresse oxidativo, a atividade deficiente do receptor de insulina, a inflamação e os danos vasculares, além de perda sináptica e morte/atrofia neuronal ${ }^{3}$. Muitas destas características também estão presentes no diabetes mellitus tipo 2 (DM2) 4 .

O DM2 pode ser caracterizado pela presença de resistência à insulina e alteração na secreção de insulina pelas células beta pancreáticas. $\bigcirc$ mecanismo inicial da doença inclui o aumento da secreção de insulina em resposta à resistência insulínica, desencadeando um estado de hiperinsulinemia. Com a progressão da doença, a função das células beta começa a declinar e a produção da insulina se torna inadequada para superar o estado de resistência a esse hormônio, resultando em hiperglicemia, a qual evolui para pré-diabetes e $\mathrm{DM} 2^{5}$.

A insulina, além de atuar no metabolismo da glicose cerebral e mobilização de GLUT4 para captação de glicose, é capaz de modificar a plasticidade sináptica, facilitar o crescimento de neuritos, ter efeitos neuroprotetores e é responsável pela modulação cognitiva ${ }^{6}$. Assim, a insulina atua controlando a liberação de neurotransmissores na atividade sináptica, estando associada à aprendizagem, memória a longo prazo, além do metabolismo energético e da sobrevivência de neurônios. Consequentemente, qualquer alteração na atividade da insulina pode causar prejuízo nos mecanismos de reparação, crescimento e diferenciação celulares e, consequentemente, desencadear processos neurodegenerativos ${ }^{7}$.

Estudos recentes têm demonstrado que, no início da DA, há redução na utilização de glicose pelo cérebro, levando ao comprometimento cognitivo. Foram descritas resistência e deficiência de insulina e insulin growth factor (IGF) - fator de crescimento semelhante à insulina - no cérebro, o que sustenta o papel importante da sinalização insulínica e de IGF na patogênese da $\mathrm{DA}^{8}$.

A conexão entre o DM2 e a DA vem sendo estudada há alguns anos mediante estudos experimentais com animais, nos quais foi possível reproduzir vários aspectos importantes da neurodegeneração. Uma das pesquisadoras pioneiras foi
Suzanne M. de La Monte que, em 2006, conseguiu demonstrar, por meio da administração intracerebral de estreptozotocina (agente antineoplásico alquilante de ocorrência natural tóxico para as células beta pancreáticas) em ratos, que ocorre neurodegeneração semelhante à encontrada na DA, caracterizando doença neuroendócrina intrínseca, causada por deficiências nos mecanismos de sinalização e na produção local de insulina e IGF. A injeção de estreptozotocina, portanto, comprovou que a DA pode ser causada, pelo menos em parte, pela resistência neuronal à insulina, isto é, diabetes cerebral ${ }^{9,10}$. Com este estudo surgiu o termo diabetes mellitus tipo 3 (DM3), para fornecer uma visão integrada dos mecanismos patogênicos potenciais compartilhados pelo DM2 e DA'1 .

O objetivo desta revisão é destacar a fisiopatologia do desenvolvimento de demência e sua relação com o DM2 e resistência à insulina, denominado DM3.

\section{MÉTODO}

Trata-se de uma revisão integrativa, com coleta de dados realizada a partir de fontes secundárias, por meio de levantamento bibliográfico. Esse método de pesquisa foi escolhido por sintetizar e incorporar evidências científicas de diversos estudos sobre determinado tema, de maneira a aprofundar o conhecimento sobre o assunto abordado ${ }^{12}$.

Para a elaboração desta pesquisa, foram realizadas as seguintes etapas: identificação do tema e definição da questão norteadora; busca na literatura e coleta dos dados; análise dos estudos inseridos nos critérios de inclusão; síntese e apresentação das evidências/construção da revisão.

Foram utilizados, para busca dos artigos, os seguintes descritores e suas combinações, em português, inglês e espanhol: doença de Alzheimer, resistência à insulina e diabetes mellitus tipo 3 .

Os critérios de inclusão definidos para a seleção dos artigos foram: artigos publicados em português, inglês ou espanhol, na íntegra, com foco na fisiopatologia do diagnóstico do DM3, publicados e indexados nos bancos de dados Medline e PubMed, nos últimos 5 anos. Os critérios de exclusão foram os artigos que não preencheram os critérios acima e/ou aqueles focados apenas no seu tratamento.

\section{RESULTADOS}

Foram encontrados 21 artigos, sendo 4 no Medline e 17 no Pubmed. Foram excluídos 9 artigos, 4 deles por não estarem disponíveis na íntegra e 5 por não se tratar da fisiopatologia do DM tipo 3.

Conforme o exposto na Tabela 1, foram selecionados 12 artigos para a discussão da presente revisão, sendo 
Tabela 1 - Estudos selecionados e analisados no estudo.

\begin{tabular}{|c|c|c|c|}
\hline Autor, Ano & Título & Periódico & Principais considerações \\
\hline $\begin{array}{l}\text { Zhang et al. }{ }^{13} \text {, } \\
2018\end{array}$ & $\begin{array}{l}\text { Diabetes mellitus and Alzheimer's } \\
\text { disease: GSK- } 3^{(1)} \text { as a potential } \\
\text { link }\end{array}$ & $\begin{array}{l}\text { Behavioural } \\
\text { Brain Research }\end{array}$ & Descreve o processo fisiopatológico da GSK-3ß no DM2 e DA \\
\hline $\begin{array}{l}\text { Vacinová et al. }{ }^{14} \text {, } \\
2017\end{array}$ & $\begin{array}{l}\text { Associations of polymorphis- } \\
\text { ms in the candidate genes for } \\
\text { Alzheimer's disease BIN1(1), CLU }{ }^{(3)} \text {, } \\
\text { CR1 } 1^{(4)} \text { and PICALM }{ }^{(5)} \text { with gestatio- } \\
\text { nal diabetes and impaired qlucose }\end{array}$ & $\begin{array}{l}\text { Molecular Biology } \\
\text { Reports - } \\
\text { Springer }\end{array}$ & $\begin{array}{l}\text { Pesquisa verificou que a distribuição de frequências genotí- } \\
\text { picas e alélicas em PICALM (rs3851179) encontrados em um } \\
\text { grupo relativamente grande de pacientes com DM gestacional } \\
\text { são semelhantes às frequências genotípicas e alélicas da DA(6) }\end{array}$ \\
\hline
\end{tabular}

Kandimalla et Is Alzheimer's disease a type 3 al. ${ }^{8}, 2016 \quad$ diabetes? A critical appraisal

Formiga et al. ${ }^{11}$, Demencia y diabetes: relación $2014 \quad$ casual o causal?

Li et al. ${ }^{5}, 2015$ Link between type 2 diabetes and Alzheimer's disease: from epidemiology to mechanism and treatment

De la Monte \& Type 3 diabetes is sporadic Wands ${ }^{15}, 2014 \quad$ Alzheimer's disease: mini-review

Ahmed et al. ${ }^{3}, \quad$ Linking insulin with Alzheimer's 2015 disease: emergence as type III diabetes

De la Monte et The 20-year voyage aboard the al. ${ }^{16}, 2018$

Walker \& Harrison $^{17}, 2015$

Jha et al. ${ }^{7}, 2015$ Impact of insulin degrading enzyme and neprilysin in Alzheimer's disease biology: characterization of putative cognates for therapeutic applications

docking at 'type 3 diabetes', environmental/exposure factors, pathogenic mechanisms, and potential treatments

Biochimica et Biophysica Acta

Medicina Clinica

Clinical Intervantions in Aging

European Neuropsychopharmacology

Neurological

Sciences

Journal of

Alzheimer's

Disease

Journal of

Alzheimer's

Disease

Shared neuropathological characteristics of obesity, type 2 diabetes and Alzheimer's disease: impacts on cognitive decline

Kapogiannis et Dysfunctionally phosphorylated al. ${ }^{18}, 2015$ type 1 insulin receptor substrate in neural-derived blood exosomes of preclinical Alzheimer's disease

Jayaraman \&

Alzheimer's disease and type 2

Pike $^{19}, 2014$

Nutrients - MDPI Journal

The FASEB Journal

Current Diabetes Reports diabetes: multiple mechanisms contribute to interactions
Descrição de todos os possíveis mecanismos bioquímicos que relacionam a DA e o DM, baseado em revisão da literatura

Revisão da associação entre DM e DA (demência) e o tratamento farmacológico do DM para um melhor controle metabólico dos pacientes e possível redução de danos cognitivos.

Revisão de evidências epidemiológicas entre DM2 e obesidade, hiperinsulinemia, síndrome metabólica e DA com foco na resistência e deficiência à insulina. $O$ estudo ainda discute sobre ensaios clínicos de tratamento

Revisão de estudos que relacionam a exposição experimental de doses de nitrosamina encontradas em alimentos processados ao comprometimento cognitivo, neurodegeneração na DA e resistência insulínica cerebral (DM3)

Descreve estudos relacionando o metabolismo da insulina prejudicado à fisiopatologia da DA. Demonstra uma cascata patológica comum entre a DA e o DM, e propõe que uma intervenção terapêutica com antidiabéticos para pacientes com DA pode ser promissora

Revisão de estudos publicados ao longo dos 20 anos de história do Journal of Alzheimer's Disease sobre os fatores relacionados à patogênese da doença de Alzheimer, associado a diversos fatores ambientais, até chegar ao conceito de DM3. Aborda sutilmente estratégias terapêuticas para reverter a cascata de neurodegeneração

O estudo apresenta o mecanismo proteolítico e regulador da degradação de amiloide- $\beta$, relacionado com o processo neurodegenerativo da DA, analisando a ação das enzimas degradantes de amiloide- $\beta$. Também apresenta agentes terapêuticos e estratégias relacionadas ao problema

Explicação de possíveis mecanismos patológicos e características semelhantes da DA e do DM2 que podem contribuir para déficit cognitivo, assim como os efeitos neurocognitivos e inflamatórios do consumo de dieta rica em lipídeos

Estudo transversal retrospectivo, comparando níveis totais de IRS-1 ${ }^{(7)}$ e suas formas fosforiladas em pacientes no estágio pré-clínico da DA ou com manifestação da doença em comparação a indivíduos cognitivamente saudáveis
Modificações na apolipoproteína E e níveis de testosterona e suas relações com DM2 e disfunção neural

(1) GSK-3 = Glicogênio sintase quinase 3; (2) BIN1 = Bridging Integrator $1 ;{ }^{(3)} \mathrm{CLU}=$ Clusterin; ${ }^{(4)} \mathrm{CR} 1$ = Tipo de receptor de complemento $1 ;{ }^{(5)}$ PICALM = Proteína de montagem de clatrina de ligação a fosfatidilinositol; ${ }^{(6)} \mathrm{DA}=$ Doença de Alzheimer; ${ }^{(7)}$ IRS-1 = Substrato do receptor de insulina. 
avaliadas 10 revisões de literatura e 2 estudos originais. Seis artigos $5,8,11,13,15,17$ exploraram fisiológica, biológica e bioquimicamente a relação celular e molecular entre a DA e a resistência à insulina, utilizando o termo DM3. Três estudos ${ }^{3,11,16}$ abordaram os possíveis mecanismos patogênicos; dois ${ }^{3,15}$ discutiram o papel da insulina no cérebro e dois ${ }^{5,16}$ mostraram fatores ambientais e evidências epidemiológicas vinculando diabetes à DA. Apenas um artigo ${ }^{17}$ citou efeitos e possíveis intervenções dietéticas para evitar neurodegeneração.

Em relação aos estudos originais, um avaliou e comparou pacientes com DA, DM2 e demência frontotemporal ${ }^{18}$; e outro associou quatro genes à DA em mulheres com diabetes gestacional, a fim de detectar relação entre esses genes e desordens no metabolismo da glicose ${ }^{14}$.

\section{DISCUSSÃO}

A insulina possui importante papel no crescimento celular no sistema nervoso central (SNC), no aprendizado e na memória. Ela atua nas sinapses nervosas, sendo responsável pelo início da formação da memória. Alguns estudos sugerem que a insulina não está envolvida apenas no metabolismo da glicose para a sobrevivência neuronal, mas também na regulação da neurotransmissão sináptica para o estabelecimento da sua plasticidade ${ }^{11}$. Alguns autores referem que as consequências da resistência à insulina no cérebro e prejuízo da sua sinalização comprometem a capacidade funcional cerebral, além da própria produção de energia, plasticidade e integridade da substância branca cerebral ${ }^{10,15}$.

Com a liberação adequada de insulina cerebral, ocorre captação e a utilização da glicose pelo cérebro. Dessa forma, a insulina secretada é capaz de estimular a memória e a cognição, por assegurar metabolismo adequado da glicose. Porém, se ocorrer hiperglicemia ou deficiência de insulina, pode ocorrer a cessação do balanço energético cerebral, ocasionando aumento do estresse oxidativo e acúmulo de produtos de glicação avançada (AGEs) que levam à geração de espécies reativas de oxigênio (ROS), gerando danos às células". Os AGEs são formados por moléculas heterogêneas que ocorrem aceleradamente no estado hiperglicêmico do diabetes, produzem superóxido e $\mathrm{H} 2 \mathrm{O} 2$, resultando em peroxidação lipídica e dano celular no cérebro. Cronicamente, níveis elevados de insulina periférica reduzem a sensibilidade à insulina na barreira hematoencefálica (BHE) e, portanto, há aumento da glicose no cérebro. Mesmo com glicose disponível no corpo, as células cerebrais não são capazes de derivar energia a partir dela, causando morte celular e redução da atividade neuronal ${ }^{8}$.

O metabolismo lipídico é outro agente que impacta negativamente o cérebro, já que o seu metabolismo desregulado pode acarretar no acúmulo de lipídios tóxicos em diversos órgãos. Esses lipídios tóxicos são então liberados na corrente sanguínea, podendo atravessar a BHE por sua característica lipossolúvel, causando inflamação, resistência à insulina e lesões neurotóxicas, com consequente neurodegeneração ${ }^{16}$.

Nos pacientes com DA, existe diminuição significativa da taxa de metabolismo da glicose, especialmente nas regiões onde ocorre o processamento de memória e da aprendizagem. No SNC, a Apo-E é produzida principalmente por astrócitos e transporta colesterol aos neurônios via receptores Apo-E. Já foi demonstrado que o alelo ApoEk4 da isoforma ApoE é fator de risco para $\mathrm{DA}^{8}$. Além desse alelo, outro estudo observou que altos níveis de peptídeo $\mathrm{C}$, parâmetro utilizado para avaliar a secreção de insulina, podem estar diretamente associados à piora da cognição, mesmo em pessoas não diabéticas ${ }^{5}$. Nos últimos anos, estudos de associação genômica revelaram 9 genes (BIN1, CR1, CLU, PICALM, MS4A4 / MS4A6E, CD2AP, CD33, EPHA1, ABCA7) que estão associados à DA ou podem influenciar o seu desenvolvimento ${ }^{8,14}$.

Além disso, a hiperinsulinemia e a resistência à insulina também podem causar morte neuronal e favorecer a formação de beta-amiloide $(A \beta)$ extracelular, originando fibrilas e agregados de placas neuríticas (Figura 1). Os oligômeros de peptídios da proteína precursora de beta-amiloide

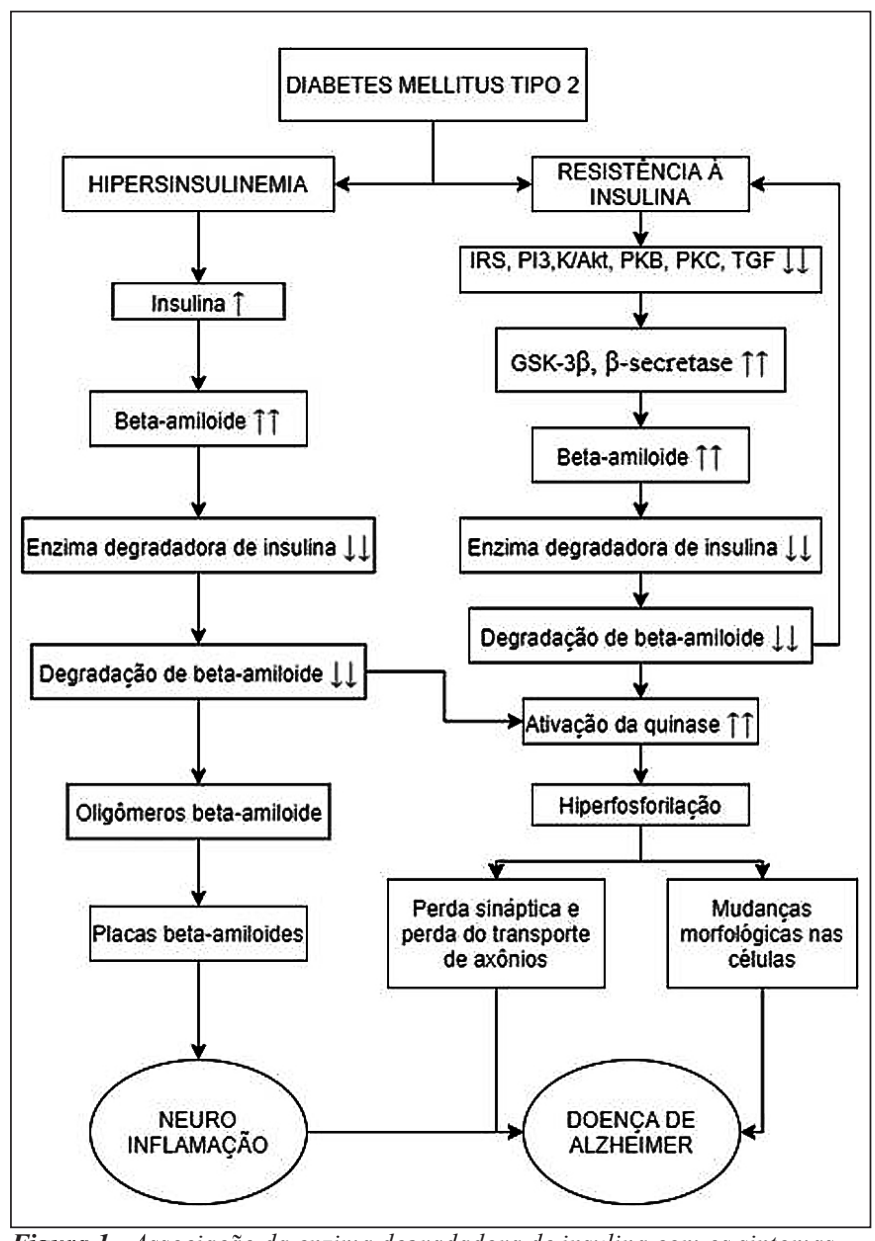

Figura 1 - Associação da enzima degradadora de insulina com os sintomas clínicos da doença de Alzheimer, assim como o risco de diabetes mellitus tipo 2 (adaptado de Jha et al..$^{7}$ ). IRS = substrato do receptor de insulina; $P I 3 \mathrm{~K} /$ $A k t=$ fosfatidilinositol 3-quinase / serina-treonina proteina quinase; $P K B=$ proteina quinase $B ; P K C=$ proteina quinase $C ; T G F=$ fator de crescimento transformador; GSK3 $\beta$ : glicogênio sintase quinase $3 \beta$ 
(AßPP-A $\beta$ ) são neurotóxicos e alteram a plasticidade da sinapse. $O$ aumento da produção de $A \beta$ dentro da célula indica depuração extracelular e faz com que $A \beta$ se acumule como placas senis. $O A \beta$ é eliminado principalmente pela insulin-degrading enzyme (IDE) - enzima degradadora de insulina ${ }^{11}$.

Em idosos, a produção de IDE diminui, havendo então uma quantidade crescente de substrato combinado com menor atividade enzimática. Da mesma forma que a insulina pode ser vista como um inibidor competitivo de IDE para degradação de $A \beta$, o $A \beta$ pode ser visto como um inibidor competitivo de insulina para o seu receptor. A degeneração prejudicada de $A \beta$ também promove a neurotoxicidade e agrava o risco de $\mathrm{DA}^{5}$.

Algumas evidências têm demonstrado que a glicogênio

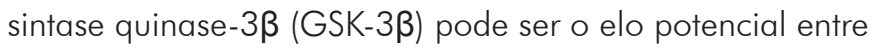
$D M$ e $D^{13}$. Já outros estudos acreditam no aumento da fosforilação da proteína Tau na resistência e deficiência de insulina mediante a ativação da GSK-3 $\beta^{5}$. No DM, a GSK- $3 \beta$ atua como enzima crucial da síntese de glicogênio, que é importante na regulação da glicemia, sendo um dos principais fatores que pode levar à deficiência de insulina e resistência à insulina. A fosforilação da GSK-3 $\beta$ pode ser ativada ou exacerbada por diversos fatores, como estresse oxidativo ou processo inflamatório, ativando uma cascata de reações que podem causar apoptose ou esgotamento das células $\beta$ pancreáticas e redução da secreção de insulina, assim eventualmente acelerando o processo de $\mathrm{DM}^{5}$.

A GSK-3ß também é uma importante enzima de fosforilação da proteína Tau e está envolvida na via de sinalização: insulina / fosfoinositídeo 3-quinase / proteína quinase B (insulina / PI3K / Akt). A disfunção desta via de sinalização pode levar à hiperfosforilação da proteína Tau. Esta proteína tem como função eliminar a deposição de $\beta$-amiloide causada pela resistência à insulina, porém devido a sua excessiva fosforilação, acaba perdendo a sua função, causando neurotoxicidade, degeneração dos neurônios, interferindo na plasticidade sináptica normal e, consequentemente, afetando as funções cognitivas ${ }^{13}$.

funcionamento prejudicado da glicose pode ser associado à regulação reduzida da OGlcNAcilação, a qual ocasiona a hiperfosforilação da proteína Tau. Além disso, a inativação da GSK-3ß, consequente à deficiência de insulina e à presença de oligômeros $A \beta$ nos neurônios, também leva ao estado de Tau anormalmente fosforilada ${ }^{3}$.

Até recentemente, acreditava-se que a captação de glicose no cérebro era inteiramente independente da insulina com os transportadores de glicose GLUT1 e GLUT3. No entanto, atualmente é reconhecido que o transportador de glicose GLUT4, receptor de insulina e transportador de glicose insulino-sensível, está presente na BHE e em alguns tipos de células cerebrais. Na DA, quando o GLUT4 ou receptores de insulina estão comprometidos, pode ocorrer hipoglicemia funcional no cérebro e, assim, diminuir a taxa de metabolismo da glicose no cérebro ${ }^{8}$.

Por fim, verificou-se que a presença do alelo $C$, que confere risco à DA, foi associada a um risco aumentado de DM gestacional. Outrossim, as pacientes com DM gestacional têm uma frequência significativamente menor do alelo T "protetor" para DA em relação ao grupo controle, e até mesmo em relação aos pacientes com DA. Esses resultados indicam que a patogênese do DM gestacional é diferente do DM2, trazendo outro importante achado relacionado ao gene PICALM, que poderia desempenhar um papel significativo na DA ${ }^{14}$.

\section{CONCLUSÃO}

Muitos fatores contribuem para a função neurocognitiva prejudicada relacionada à progressão da DA no cérebro, como o metabolismo alterado da insulina, juntamente com a expressão de determinados genes e o metabolismo dos lipídios. Os estudos envolvendo a relação entre DM2 e DA, que originaram o termo "diabetes mellitus tipo 3", são recentes, e mais pesquisas são necessárias com o intuito de aprofundar o conhecimento desta condição. $O$ melhor conhecimento da fisiopatologia poderá oferecer oportunidade de se desenvolver tratamentos que evitem a progressão da doença, melhorando a qualidade de vida dos pacientes.

\section{REFERÊNCIAS}

1. Chatterjee S, Peters SA, Woodward M, Arango SM, Batty GD, Beckett N, et al. Type 2 diabetes as a risk factor for dementia in women compared with men: a pooled analysis of 2.3 million people comprising more than 100,000 cases of dementia. Diabetes Care. 2016;39(2):300-7.

2. World Health Organization and Alzheimer's Disease International. Dementia: a public health priority. Alzheimer's Disease International, 2015. [cited $2020 \mathrm{Sep} 3$ ]. Available from: https:// apps.who.int/iris/bitstream/handle/10665/75263/9789241564 458_eng.pdf? sequence $=1$

3. Ahmed S, Mahmood Z, Zahid S. Linking insulin with Alzheimer's disease: emergence as type III diabetes. Neurol Sci. 2015;36(10):1763-9.

4. Greene A, Ng J, Sheperd L, Carey K. Alzheimer's disease and type 2 diabetes: what is the connection? Consult Pharm. 2015;30(2):112-5.

5. Li X, Song D, Leng SX. Link between type 2 diabetes and Alzheimer's disease: from epidemiology to mechanism and treatment. Clin Interv Aging. 2015;10:549-60.

6. Al Haj Ahmad RM, Al-Domi HA. Thinking about brain insulin resistance. Diabetes Metab Syndr. 2018;12(6):1091-4.

7. Jha NK, Jha SK, Kumar D, Kejriwal N, Sharma R, Ambasta $\mathrm{RK}$, et al. Impact of insulin degrading enzyme and neprilysin in alzheimer's disease biology: characterization of putative cognates for therapeutic applications. J Alzheimers Dis. 2015;48(4):891-917.

8. Kandimalla R, Thirumala V, Reddy PH. Is Alzheimer's disease a type 3 diabetes? A critical appraisal. Biochim Biophys Acta Mol Basis Dis. 2017;1863(5):1078-89. 
9. de la Monte SM, Wands JR. Review of insulin and insulin-like growth factor expression, signaling, and malfunction in the central nervous system: relevance to Alzheimer's disease. J Alzheimers Dis. 2005;7(1):45-61.

10. de la Monte SM, Tong M, Lester-Coll N, Plater M Jr, Wands JR. Therapeutic rescue of neurodegeneration in experimental type 3 diabetes: relevance to Alzheimer's disease. J Alzheimers Dis. 2006;10(1):89-109.

11. Formiga F, Pérez-Maraver M. Type 3 diabetes mellitus. The revival of inhaled insulin? Endocrinol Nutr. 2014;61(4):173-5.

12. Mendes KDS, Silveira RCCP, Galvão CM. Revisão integrativa: método de pesquisa para a incorporação de evidências na saúde e na enfermagem. Texto Context - Enferm. 2008;17(4):758-64.

13. Zhang Y, Huang NQ, Yan F, Jin H, Zhou SY, Shi JS, et al. Diabetes mellitus and Alzheimer's disease: GSK-3 $\beta$ as a potential link. Behav Brain Res. 2018;339:57-65.

14. Vacínová G, Vejražková D, Lukášová P, Lischková O, Dvořáková $\mathrm{K}$, Rusina $\mathrm{R}$, et al. Associations of polymorphisms in the candidate genes for Alzheimer's disease BIN1, CLU, CR1 and
PICALM with gestational diabetes and impaired glucose tolerance. Mol Biol Rep. 2017;44(2):227-31.

15. de la Monte SM, Wands JR.Alzheimer's disease is type 3 diabetesevidence reviewed. J Diabetes Sci Technol. 2008;2(6):1101-13.

16. de la Monte SM, Tong M, Wands JR. The 20-year voyage aboard the journal of Alzheimer's disease: docking at 'type 3 diabetes', environmental/exposure factors, pathogenic mechanisms, and potential treatments. J Alzheimers Dis. 2018;62(3):1381-90.

17. Walker JM, Harrison FE. Shared neuropathological characteristics of obesity, type 2 diabetes and Alzheimer's disease: impacts on cognitive decline. Nutrients. 2015;7(9):7332-57.

18. Kapogiannis D, Boxer A, Schwartz JB, Abner EL, Biragyn A, Masharani U, et al. Dysfunctionally phosphorylated type 1 insulin receptor substrate in neural-derived blood exosomes of preclinical Alzheimer's disease. FASEB J. 2015;29(2):589-96.

19. Jayaraman A, Pike CJ. Alzheimer's disease and type 2 diabetes: multiple mechanisms contribute to interactions. Curr Diab Rep. 2014;14(4):476.

Local de realização do estudo: Universidade Federal do Paraná, Curitiba, PR, Brasil.

Conflito de interesse: Os autores declaram não haver. 